Ihr Patient will sportlich aktiv werden

\title{
Drohen ihm gefährliche Rhythmusstörungen?
}

\begin{abstract}
Bei sportlicher Betätigung treten arrhythmiebedingte kardiale Zwischenfälle häufiger auf als ohne Sport. Deshalb wird der Arzt gelegentlich von Patienten, die ein Training beginnen wollen, um eine Abschätzung ihres Risikos gebeten. Besonderer Aufmerksamkeit bedürfen Personen, die früher intensiv Sport getrieben haben und nach einer längeren Pause wieder einsteigen. Auch beim Sport hängt die Gefährlichkeit von Arrhythmien wesentlich davon ab, ob eine strukturelle Herzkrankheit besteht.
\end{abstract}

- Herzrhythmusstörungen bei Sportlern sind in der Regel seltene Ereignisse und meist harmlos. Als prädisponierende Faktoren spielen Elektrolytstörungen (Hyponatriämie), Umweltbedingungen (Hitze, Kälte, Schadstoffe), QT-verlängernde Medikamente, anabole Steroide, Drogen oder Alkohol eine Rolle. Anhand von neuen EKG-Kriterien lässt sich heute besser unterscheiden, was für den Sportler gefährlich und was ungefährlich ist, erklärte Prof. Herbert Löllgen, niedergelassener Kardiologe und Sportmediziner in Remscheid.

\section{Harmlose EKG-Veränderungen}

Häufige trainingsbedingte, aber harmlose Veränderungen sind einzelne Extrasystolen, Sinusbradykardie, AV-Block 1. Grades mit inkomplettem Rechtsschenkelblock, frühe Repolarisation, Linkshypertrophie-Zeichen und negative T-Wellen bis V3. Aus Langzeit-EKG-Untersuchungen geht hervor, dass etwa ein Fünftel der hochtrainierten Sportler während körperlicher Belastung ventrikuläre Extrasystolen haben. Ob die Extrasystolen in Ruhe oder bei Belastung auftreten, hat keine diagnostische Wertigkeit. Verursachen die genannten Arrhythmien keine Symptome, bedeuten sie keine Einschränkung für die sportliche Aktivität.

\section{EKG-Befunde, die ein Risiko signalisieren}

Synkopen bei einem Sportler müssen dagegen immer genau abgeklärt werden. Als anormal muss betrachtet werden, wenn in Zusammenhang mit sportlicher Betätigung über fünf supraventrikuläre Extrasystolen hintereinander beobachtet werden oder ein paroxysmales Vorhofflimmern bzw. eine paroxysmale supraventrikuläre Tachykardie auftritt. Abnormal sind auch komplexe ventrikuläre Arrhythmien, häufige ventrikuläre Extrasystolen ( $>5 / \mathrm{min}$, Salven, Couplets).

An kardiale pathologische Prozesse muss man denken bei negativen T-Wellen über V3 hinaus (hypertrophe obstruktive Kardiomyopathie), ST-Senkung (Koronargefäßerkrankung), pathologischen Q-Zacken (linksatriale Vergrößerung), Zeichen für Rechtsherzhypertrophie und komplettem Rechtsoder Linksschenkelblock.

Bei jüngeren Menschen mit einem totalen AV-Block muss man immer an eine Borreliose oder Sarkoidose denken, betonte Löllgen. Auch eine Verlängerung der absoluten und relativen QTDauer oder der QT-Dispersion während Belastung wird als pathologisch angesehen. Als Ursache kommen QT-verlängernde Medikamente wie Antihistaminika und Makrolide in Betracht. Bedrohlich sind auch das BrugadaSyndrom und Präexzitationssyndrome.

\section{Mit Rhythmuskontrolle mehr Sicherheit beim Sport}

Ventrikuläre Extrasystolen bedürfen in der Regel keiner Therapie. Bei hartnäckigen häufigen ventrikulären Extrasystolen kann aber gelegentlich eine Ablation indiziert sein. Bei der Wahl einer

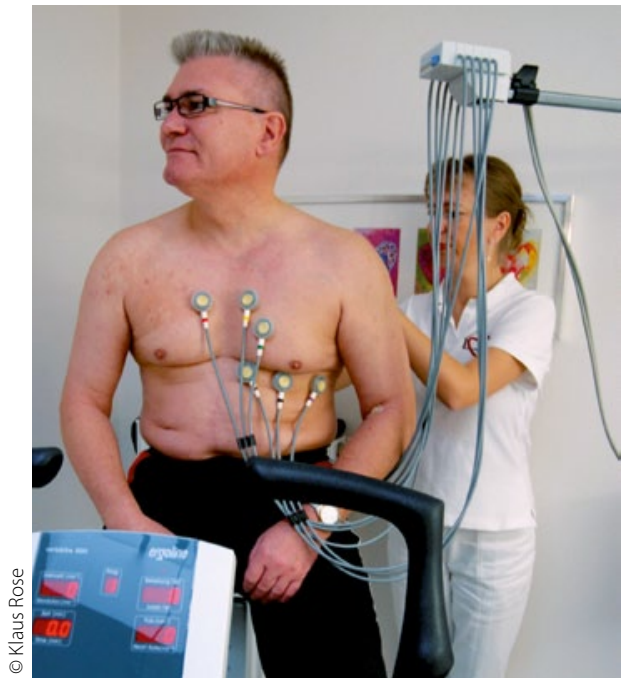

Gefährdet er durch Sport sein Herz? Ein Belastungs-EKG kann Risiken aufdecken.

medikamentösen Therapie muss bedacht werden, dass Ausdauersportler Betarezeptorenblocker oft nicht gut vertragen.

Seltene intermittierend auftretende Arrhythmien kann der Patient durch die "pill in the pocket" Therapie kontrollieren. Bei häufigen Episoden von supraventrikulären Arrhythmien ist eine Ablation zu überlegen. Dies gilt besonders für das WPW-Syndrom, wo diese Indikation heute eher großzügig gestellt wird. „Wir haben in unserer Klinik sechs Sportler mit Herzstillstand aufgrund eines WPWSyndroms mit intermittierendem Vorhofflimmern erfolgreich wiederbelebt", so Löllgen. Ein intermittierendes oder neu aufgetretenes Vorhofflimmern kann auch durch eine Pulmonalvenenablation häufig beherrscht werden.

\section{Sport mit Schrittmacher?}

Bei komplexen ventrikulären Arrhythmien kann ein Defibrillator nötig werden. Auch Patienten mit Rechtsschenkelblock oder Brugadasyndrom brauchen einen AICD. Schrittmacherträger sollten Sportarten mit starker Belastung im Schulterbereich meiden. Auch auf Sportarten, bei denen sie sich oder andere durch einen Schock mit kurzdauerndem Bewusstseinsverlust gefährden können, sollten sie verzichten.

\section{DR. MED. ANGELIKA BISCHOFF}

- 118. Kongress der Deutschen Gesellschaft für Innere Medizin, Wiesbaden, 14.-17. April 2012 